\title{
Extension of Equivalent System Mass for Human Exploration Missions on Mars
}

\author{
Aaron J. Berliner ${ }^{1,2, *}$, George Makrygiorgos ${ }^{1,3}$, and Avery Hill ${ }^{1,2}$ \\ ${ }^{1}$ Center for the Utilization of Biological Engineering in Space (CUBES), http: / / cubes . space / \\ ${ }^{2}$ Department of Bioengineering, University of California Berkeley, Berkeley, CA \\ ${ }^{3}$ Department of Chemical and Biomolecular Engineering, University of California Berkeley, Berkeley, CA \\ *e-mail: aaron.berliner@berkeley.edu
}

\begin{abstract}
NASA mission systems proposals are often compared using an equivalent system mass (ESM) framework wherein all elements of technology to deliver an effect- its components, operations and logistics of delivery- are converted to effective masses since this has a known cost scale in space operations. To date, ESM methods and the tools for system comparison have not considered complexities wherein systems that serve a mission span multiple transit and operations stages, such as would be required to support a crewed mission to Mars, and thus do not account for the different mass equivalency factors operational during each period and the inter-dependencies of the costs across the mission. Further, ESM does not account well for the differential reliabilities of the underlying technologies. Less reliability should incur an equivalent mass cost for technologies that might otherwise provide a mass advantage. We introduce an extensions to ESM to address these limitations and show that it provides a direct method for analyzing, optimizing and comparing different mission systems. We demonstrate our extended ESM (xESM) calculation with crop production technologies - an aspect of the developing offworld biomanufacturing suite since it represents a case with strong coupling among stages of the mission and a relatively high-risk profile.
\end{abstract}

Keywords: Space Systems Bioengineering, Equivalent System Mass, ESM, Environmental Control and Life Support Systems, ECLSS, Space Logistics, Uncertainty, Risk

\section{Introduction}

Travel to space is limited by the expense of transporting resources beyond the Earth's gravity well ${ }^{1}$. As a result, early metrics of usability for space systems, especially life support ${ }^{2}$, were based on mass as the primary factor. Following a request to "provide the designers of future missions with mature technologies and hardware designs, as well as extensive performance data justifying confidence that highly reliable Advanced Life Support Systems (ALS) that meet mission constraints can be developed" by the 1997 NASA Research Council (NRC) ${ }^{3}$, the scope of the Equivalent System Mass (ESM) framework was broadened to account for differences in the cost of resources ${ }^{4}$. The general principle behind this early metric was to calculate the mass of all of the resources required to make the system work ${ }^{5}$. ESM was expanded from theory ${ }^{6}$ to the practice of accounting for processes ranging from controls ${ }^{7}$, agriculture ${ }^{8}$, and recycling ${ }^{9}, 10$. Currently, ESM remains the standard metric for evaluating ALS technology development ${ }^{9,11,12}$ and systems ${ }^{13-16}$. It has been adopted for use in trade studies ${ }^{17-19}$, as the the metric for life support sizing ${ }^{20-22}$, and has been incorporated into several tools ${ }^{23-25}$.

In its current form ${ }^{26}$, the total ESM $\mathfrak{M}$ is defined only for the operations at a specific location as the sum over the set of all systems as

$$
\mathfrak{M}=L_{\text {eq }} \sum_{i=1}^{\mathcal{A}}[\underbrace{\left(M_{i} \cdot M_{\mathrm{eq}}\right)+\left(V_{i} \cdot V_{\mathrm{eq}}\right)+\left(P_{i} \cdot P_{\mathrm{eq}}\right)+\left(C_{i} \cdot C_{\mathrm{eq}}\right)}_{\mathfrak{M}_{\mathrm{NCT}}}+\underbrace{\left(T_{i} \cdot D \cdot T_{e q}\right)}_{\mathfrak{M}_{\mathrm{CT}}}]
$$

for subsystem $i \in \mathcal{A}$ of the ESM excluding crew-time $\mathfrak{M}_{\mathrm{NCT}}$ and the ESM including crewtime $\mathfrak{M}_{\mathrm{CT}}$ where $M_{i}, V_{i}, P_{i}, C_{i}$ are the initial mass $[\mathrm{kg}]$, volume $\left[\mathrm{m}^{3}\right]$, power requirement $\left[\mathrm{kW}_{e}\right]$, and cooling requirement $\left[\mathrm{kg} / \mathrm{kW}_{\mathrm{th}}\right], D$ is the duration of the mission segment [sol], $T_{i}$ is the crew-time requirement based on an astronaut crew-member $(\mathrm{CM})[\mathrm{CM}-\mathrm{h} / \mathrm{sol}], M_{\mathrm{eq}}$ is the stowage factor for accounting for additional structural masses for a subsystem such as shelving $[\mathrm{kg} / \mathrm{kg}], V_{\text {eq }}$ is the mass equivalency factor for the pressurized volume support infrastructure $\left[\mathrm{kg} / \mathrm{m}^{3}\right], P_{\mathrm{eq}}$ is the mass equivalency factor for the power generation support infrastructure $\left[\mathrm{kg} / \mathrm{kW}_{e}\right], C_{e q}$ is the mass equivalency factor for the cooling infrastructure $[\mathrm{kg} / \mathrm{kW}$ th $], T_{\mathrm{eq}}$ is the mass equivalency factor for the crew-time $[\mathrm{kg} / \mathrm{CM}-\mathrm{h}]$, and $L_{\mathrm{eq}}$ is the location factor for the mission segment $[\mathrm{kg} / \mathrm{kg}]$ 
which accounts for the cost to transport mass from one location in space to another (such as Earth orbit to Martian orbit). Mass equivalency factors $\left(V_{\mathrm{eq}}, P_{\mathrm{eq}}, C_{\mathrm{eq}}, T_{\mathrm{eq}}\right)$ are used to convert the non-mass parameters to mass.

While the ESM framework ${ }^{26}$ has been widely adopted in Environmental Control and Life Support Systems (ECLSS) analysis ${ }^{24,27-31}$, it has faced critique for the ambiguity in its application, an inability to account for development costs ${ }^{32}$, and most recently, failure to account for uncertainty ${ }^{33}$. Alternative frameworks have been proposed to replace ${ }^{34}$ or extend ESM with additional metrics ${ }^{35}$ that factor in complexity ${ }^{36}$. Given the widespread use of ESM, we believe that the framework should be improved with the addition of missing elements rather than replaced completely.

Previous efforts to quantify the cost in problems of space logistics rely on a metric based solely on the Initial Mass to Low Earth Orbit (IMLEO) $)^{37}$ for constant commodity supply and demand ${ }^{38}$. In such logistics frameworks like SpaceNet ${ }^{39-41}$ and $\mathrm{HabNet}^{42}$, cost is kept simple to allow for the analysis of complex mission architectures with multiple mission segments. Comparatively, ESM has been most fully developed for ECLSS where the costs of capital equipment, power, operations, transport and other things have been captured on a common unit scale of mass. While providing a method for summing the weighted terms of many subsystems, there is no explicit ESM equation that captures total mission cost across systems in various stages of a complex mission ${ }^{32}$. Thus the standard ESM approach is not valid as there (1) exists no explicit language for capturing the set of all segments and (2) there exists interdependent relationships between the decision variables within separate segments. Here we see a tradeoff in the complexity of the cost function for the complexity of the mission architecture.

As plans for human exploration continue to be made in anticipation of the return to the moon ${ }^{43,44}$ and onto Mars ${ }^{45}$, an added emphasis will be required on optimization of mission architecture ${ }^{41}$. As of now, the current instance of the ESM framework does not lend itself for use as an objective function in an optimization over a mission. The result is that this standard framework remains fixed for multi-stage missions and generally (but not always ${ }^{28}$ ) fails to provide design or planning information based on subsystem risk, and thus fails in its purpose to compare missions with differential reliability for systems in their proper context. That is, given two possible technologies for meeting a mission objective, the one that is less likely to fail might be a better choice. So to demonstrate how to formally add reliability metrics to the ESM framework we take the case of a new technology platform, biomanufacturing ${ }^{46-48}$, for which there are known and quantifiable reliability concerns and for which there is little in situ testing for space missions. In the following work, we propose an extend ESM (xESM) framework to account for the proposed multi-stage missions and critical mission features such as reliability. As the scope of human exploration missions has expanded, the need for new technology platforms has grown and it has been proposed that these features best capture the potential of biomanufacturing systems ${ }^{48}$. We do not claim a completion of $\mathrm{xESM}$, but rather, we demonstrate progress along this trajectory in the form of a rigorous mathematical framework to (1) account for multi-staged mission segments (beyond simple summation); (2) account for reliability; and (3) feed into downstream optimization problems.

\section{Extending ESM for Long-Duration Mission Scenarios}

Figure 1 depicts three scenarios with varied transit architecture. Scenario 1 (grey) uses a single journey from Earth to Mars, and although it has been proposed in some forms ${ }^{49}$, it is unlikely this architecture will be adopted due large mass requirements by the transit ship and the related large mass of ascent propellant required to leave Mars ${ }^{50}$. In the case of Scenario 2 (blue), cargo can be predeployed to Mars through some number of predeployment missions. Scenario 2 introduces the segments to a crewed mission to Mars which are not actually crewed, but instead either purely cargo-based in which only the $M$ and $V$ terms factor into the ESM cost, or autonomous where $M, V, P$ and $C$ for uncrewed operations matter. Since cargo missions do not require life support systems, the $M$ cost is reduced greatly ${ }^{13}$, leading to a reduction in overall mission cost, especially for missions that require a great quantity of goods that can be predeployed. In the more likely Scenario $3^{51,52}$, the crew transportation further broken down such that smaller crewed vehicles make the jump from planet to surface and vice-versa, but the interplanetary transit is made on a larger craft to reduce the mass required for egress from planetary gravity-wells.

Previous ESM literature allows for varied equivalency factors based on mission staging ${ }^{26}$, and in such cases, the ESM of distinct segments of a mission are calculated separately, then normalized through the use of location factors ${ }^{54}$. However, ESM $\mathfrak{M}$ for any set of systems is calculated using a single location factor $L_{\mathrm{eq}}$ term as a multiplier. In this form it is assumed that each subsystem is transported in uniform fashion or that all parts of a subsystem would correspond to a single $L_{\text {eq }}$ term. The scenario expansion in Figure shows that inventory can be transported in different segments using different crafts which change the value of $L_{\text {eq }}$. This is supported by non-ESM logistics methods ${ }^{41}$. We argue that the use of predeployment missions for transporting cargo nullifies the assumption. Furthermore, we begin the articulation of xESM with a mathematical formalism for multi-segmented missions.

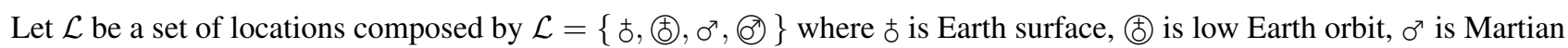
Surface, and $(0)$ is low Martian orbit. Let $\mathcal{L}_{2}$ be the set of pairs in $\mathcal{L}$ which describe from starting to ending location. Let 


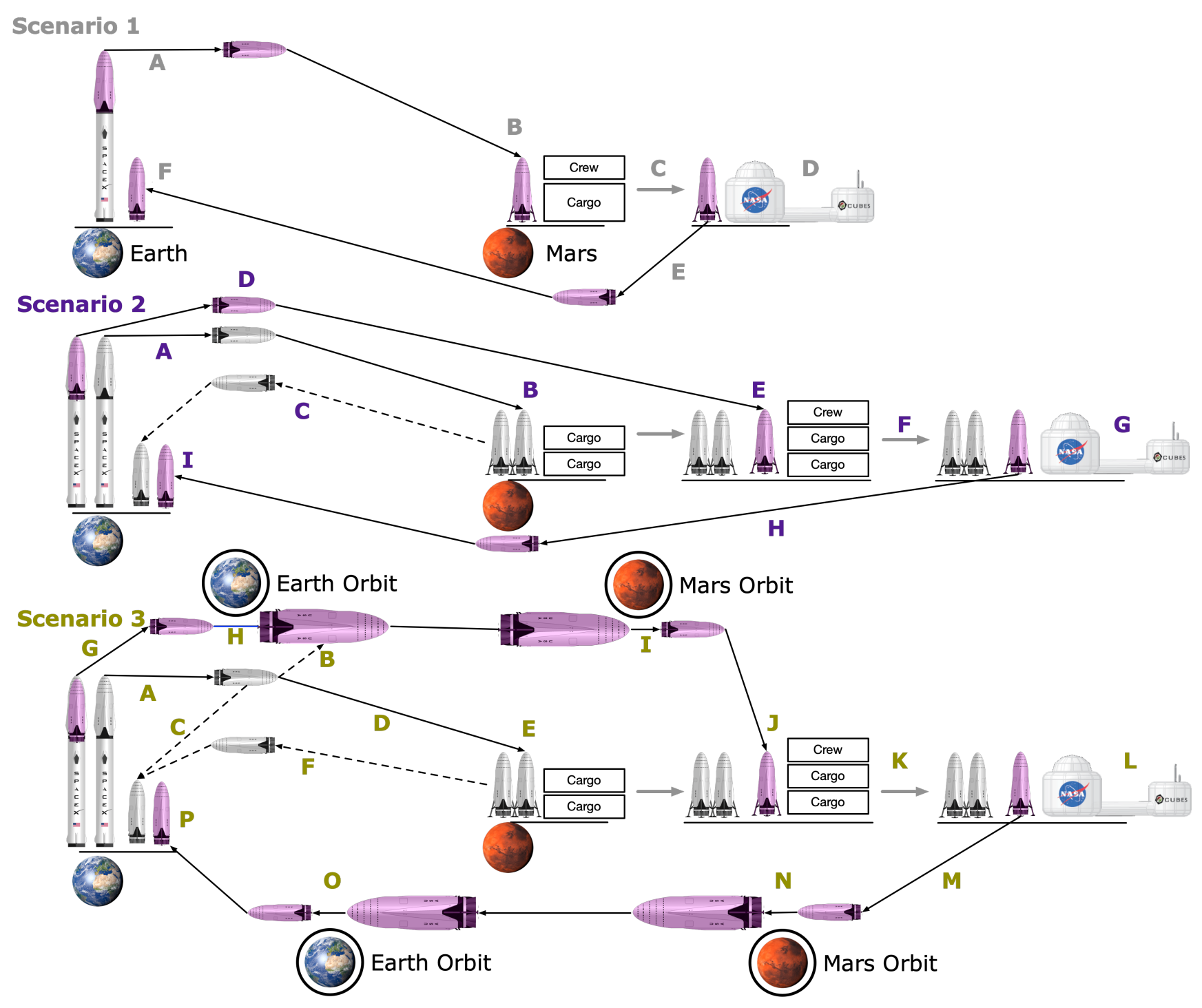

Figure 1. Transit Diagram of proposed Mission Architecture. In Scenario 1(grey), (A) a crewed transit ship is launched directly from the surface of Earth and (B) lands on the surface of Mars where (C) the crew assembles the cargo in a habitat and carries out (D) surface operations until (E) the crew launches from their initial transit ship from the surface of Mars into space and (F) lands back on the surface of Earth. In Scenario 2(purple), (A) cargo transit ships without crew are launched directly from the surface of Earth and (B) land on the surface of Mars where cargo can be unloaded. In the case of reusable rocket systems $^{53}$, (C) the cargo rockets can be launched from Mars and returned to Earth. Once all the cargo has been loaded on the surface of Mars, (D) a crewed transit ship is launched directly from the surface of Earth and (E) lands on the surface of Mars where $(\mathrm{F})$ the crew assembles the cargo in a habitat and carries out $(\mathbf{G})$ surface operations until $(\mathbf{H})$ the crew launches from their initial transit ship from the surface of Mars into space and (I) lands back on the surface of Earth. In Scenario 3(green), a number of (A) cargo transit ships without crew are launched directly from the surface of Earth and either (B) supply a previously interplanetary rocket then (C) return to the surface of Earth or (D) travel to the surface of Mars where (E) cargo can be unloaded. In the case of reusable rocket systems, (F) the cargo rockets can be launched from Mars and returned to Earth. Once all the cargo has been loaded on the surface of Mars, (G) a crewed transit ship is launched directly from the surface of Earth to Earth Orbit $\mathbf{( H )}$ where it rendezvous with an interplanetary rocket which (I) travels to Martian orbit. The crew $(\mathbf{J})$ then boards a descent vehicle and lands on the surface of Mars where (K) the crew assembles the cargo in a habitat and carries out (L) surface operations until (M) the crew launches from their initial transit ship from the surface of Mars into (N) Martian orbit where they again rendezvous with their interplanetary rocket which travels to $(\mathbf{O})$ Earth orbit at which point they board a descent rocket in which they $(\mathbf{P})$ finally return to the surface of Earth. 
$\mathcal{O}$ be the set of operations composed by $\mathcal{O}=\{F, B, A\}$ where $F^{1}$ is cargo, $B^{2}$ is robotic, and $\AA^{3}$ is crewed. Let $\Lambda(i, j)$ be the mapping from some pair of $i \in \mathcal{L}_{2}, j \in \mathcal{O}$ to the set $\mathcal{R}$ of rockets, vehicles, and habitats. A mission segment $\mathcal{S}$ can be constructed via set-builder notation as $\mathcal{S}=\left\{(i, j) \mid i \in \mathcal{L}_{2} ; j \in \mathcal{O}\right\}$ for specific combinations of locations and operations as

$$
\begin{aligned}
& \mathcal{S}=\left\{(i, j) \mid i \in \mathcal{L}_{2} ; j \in \mathcal{O}\right\}
\end{aligned}
$$

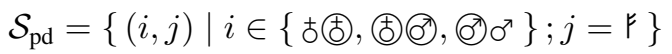

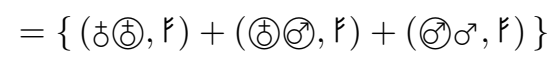

$$
\begin{aligned}
& \mathcal{S}_{\mathrm{sf}}=\left\{(i, j) \mid i=\sigma^{\lambda} \sigma^{\top} ; j \neq \mathrm{F}\right\} \\
& =\left\{\left(\sigma^{x} \sigma^{x}, B\right)+\left(\sigma^{x} \sigma^{x}, A\right)\right\}
\end{aligned}
$$

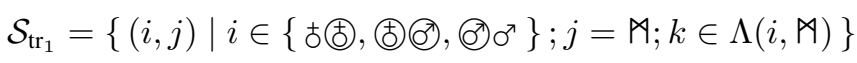

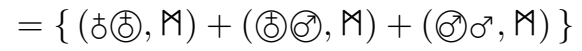

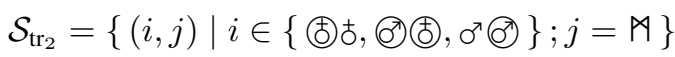

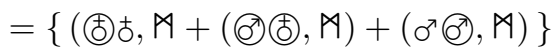

$$
\begin{aligned}
& \mathcal{S}_{\mathrm{tr}_{3}}=\{(i, j) \mid i \in\{(0)(0)\} ; j \neq \mathrm{F}\} \\
& =\left\{\left(O^{\circ}(0), B\right)+\left(\theta^{\circ}\left(\theta^{\circ}, A\right)\right\}\right.
\end{aligned}
$$

for the abstract segments of predeployment (pd), crewed transit from Earth to Mars $\left(\operatorname{tr}_{1}\right)$, Martian surface operations (sf), crewed transit back from Mars to Earth $\left(\operatorname{tr}_{2}\right)$, and either autonomous or crewed operations aboard the interplanetary vehicle in Martian orbit $\left(\operatorname{tr}_{3}\right)$. The complete mission object $\mathcal{M}$ is therefore constructed as the collection of these abstract segments in conjunction with the selection of a specific technology in $\mathcal{R}$ as

$$
\mathcal{M}=\left\{(k, \ell) \mid k=(i, j) \forall\left\{\mathcal{S}_{\mathrm{pd}}, \mathcal{S}_{\mathrm{sf}^{\mathrm{f}}}, \mathcal{S}_{\mathrm{tr}_{1}}, \mathcal{S}_{\mathrm{tr}_{2}}, \mathcal{S}_{\mathrm{tr}_{3}}\right\} ; \ell=\Lambda(i, j)\right\}
$$

and can be used in the construction of a generalized total mission ESM $\mathfrak{M}_{0}$ as

$$
\begin{aligned}
\mathfrak{M}_{0} & =\sum_{k}^{\mathcal{M}} \underbrace{L_{\mathrm{eq}, k} \sum_{i}^{\mathcal{A}_{k}}\left[\left(M_{k_{i}} \cdot M_{\mathrm{eq}, k}\right)+\left(V_{k_{i}} \cdot V_{\mathrm{eq}, k}\right)+\left(P_{k_{i}} \cdot P_{\mathrm{eq}, k}\right)+\left(C_{k_{i}} \cdot C_{\mathrm{eq}, k}\right)+\left(T_{i} \cdot D_{k} \cdot T_{\mathrm{eq}, k}\right)\right]}_{\mathfrak{M}_{0, k}} \\
& =\mathfrak{M}_{0, \mathrm{pd}}+\mathfrak{M}_{0, \mathrm{~s}}+\mathfrak{M}_{0, \mathrm{tr}_{1}}+\mathfrak{M}_{0, \mathrm{tr}_{2}}+\mathfrak{M}_{0, \mathrm{r}_{3}}
\end{aligned}
$$

as the sum of ESM for segments in a mission set $\mathcal{M}$. Essentially, we have established a graph where the locations represent nodes and the segments represent arcs which matches previous formulations of mission logistics ${ }^{41}$, although our set of location nodes is reduced for simplicity and does not include specific Lagrange Points ${ }^{37}$. The generalization enables accounting of mission segment specific terms such as location factor $L_{\mathrm{eq}}$ and equivalency factors ( $M_{\mathrm{eq}}, V_{\mathrm{eq}}, P_{\mathrm{eq}}, C_{\mathrm{eq}}, T_{\mathrm{eq}}$ ). This generalization also allows for indexing of mission segment specific subsystems $\mathcal{A}$, further enabling an accounting of inventory elements between mission segments.

xESM does not include recent developments in resupply logistics ${ }^{56}$ as enabled by the decreasing cost to LEO $^{57}$. Since these developments have been primarily applied to longer-duration ECLSS systems for the international space station (ISS) and not Mars missions. Despite a decreased cost to LEO, resupply logistics will be unlikely to impact the initial set of crewed exploration missions ${ }^{50}$ given the difference in resupply costs between the $\delta(\delta)$ and $\delta \sigma^{7}$ systems. Despite early arguments against the adoption of crew-time within the $\mathrm{ESM}^{58}$, we include these terms in our formulation as it has been the standard.

\section{Dependent Factors}

Despite now having a usable method for indexing factors by their location, operation, and hardware, the ESM framework does not account for relationships between equivalency/location factors and the segment inventory that defines them. In essence, equivalency/location factors convert non-mass properties to mass properties by means of a ratio, but because that mass originates from some subset of inventory elements, equivalency and location factors are coupled. The exact nature of this interaction depends on the scenario and the modeling itself, and aim to present a preliminary rendering of these relationships in Figure 2 by the colored markers denoting dependencies of systems elements below terms in the expanded ESM formulation. The color-coded expansion in Figure 2 includes a breakdown of components and their relationship to the terms in the equation

\footnotetext{
${ }^{1}$ Elder Furthark ${ }^{55}$ rune $F *$ fehu meaning "cattle", used here to imply "cargo"

${ }^{2}$ Elder Furthark rune B *berkanan meaning "tree", used here to imply "autonomy"

${ }^{3}$ Elder Furthark rune $\AA^{*}$ *mannaz meaning "man", used here to imply "crewed"
} 


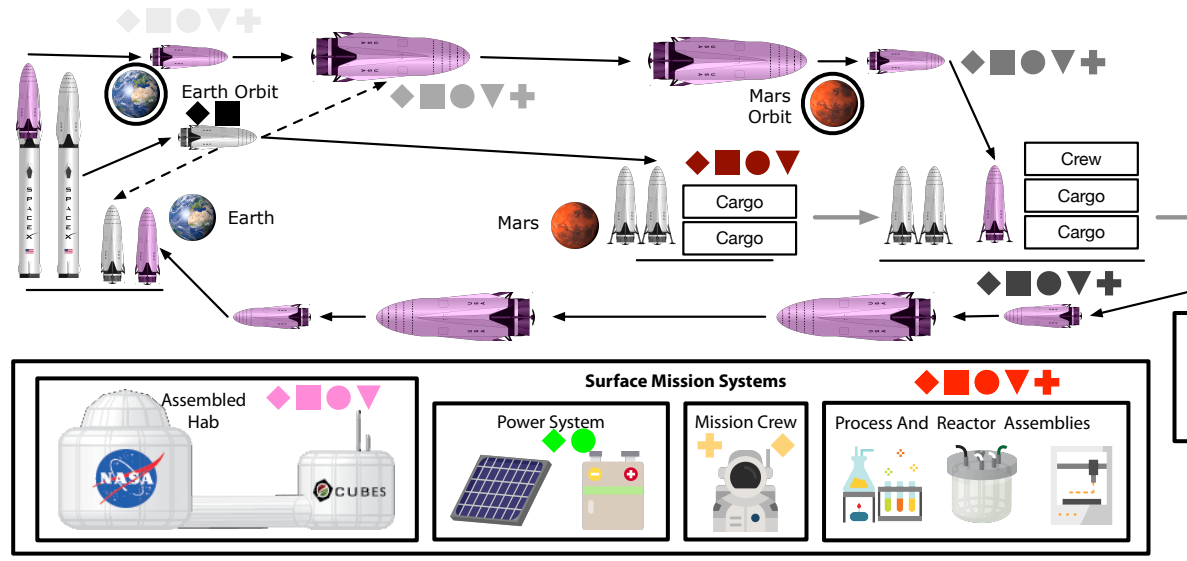

$\mathfrak{M}_{0}=\sum_{k}^{\mathcal{M}} \underbrace{L_{\mathrm{eq}, k} \sum_{i}^{\mathcal{A}_{k}}\left[\left(M_{k_{i}} \cdot M_{\mathrm{eq}, k}\right)+\left(V_{k_{i}} \cdot V_{\mathrm{eq}, k}\right)+\left(P_{k_{i}} \cdot P_{\mathrm{eq}, k}\right)+\left(C_{k_{i}} \cdot C_{\mathrm{eq}, k}\right)+\left(T_{i} \cdot D_{k} \cdot T_{\mathrm{eq}, k}\right)\right]}_{\mathfrak{M}_{0, k}}$

$=\mathfrak{M}_{0, \mathrm{pd}}+\mathfrak{M}_{0, \mathrm{sf}}+\mathfrak{M}_{0, \mathrm{tr}_{1}}+\mathfrak{M}_{0, \mathrm{tr}_{2}}+\mathfrak{M}_{0, \operatorname{tr}_{3}}$

$=\sum_{j=1}^{n} L_{\mathrm{eq}, \mathrm{pd}_{j}} \sum_{i}^{\mathcal{A}_{\mathrm{pd}_{j}}}\left[\left(M_{\mathrm{pd}_{j i}} \cdot M_{\mathrm{eq}_{,} \mathrm{pd}_{j}}\right)+\left(V_{\mathrm{pd}_{j i}} \cdot V_{\mathrm{eq}, \mathrm{pd}_{j}}\right)\right]+\ldots$

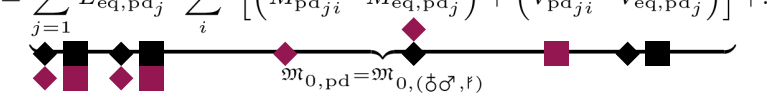

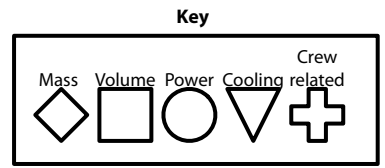

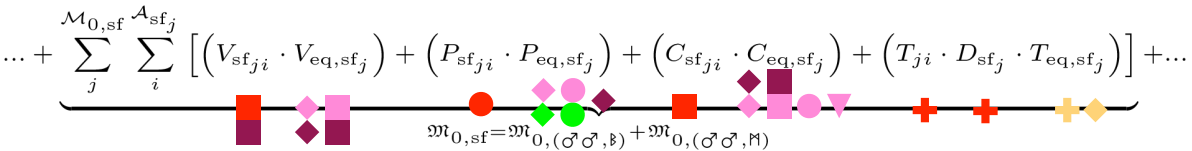

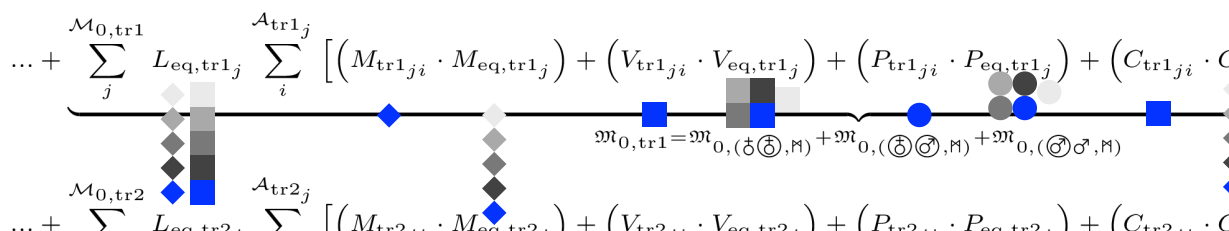

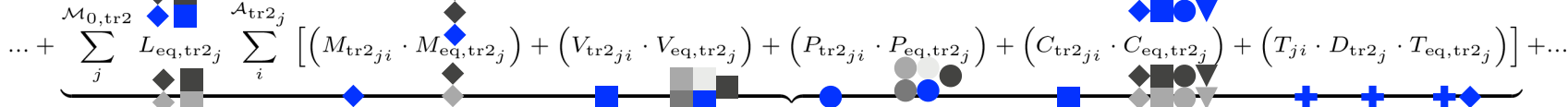

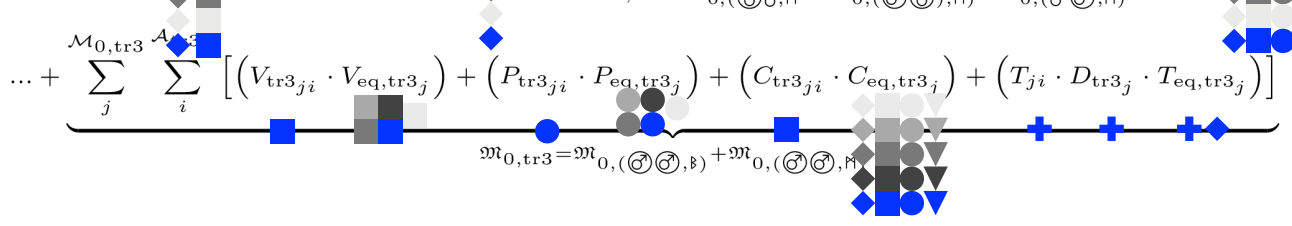

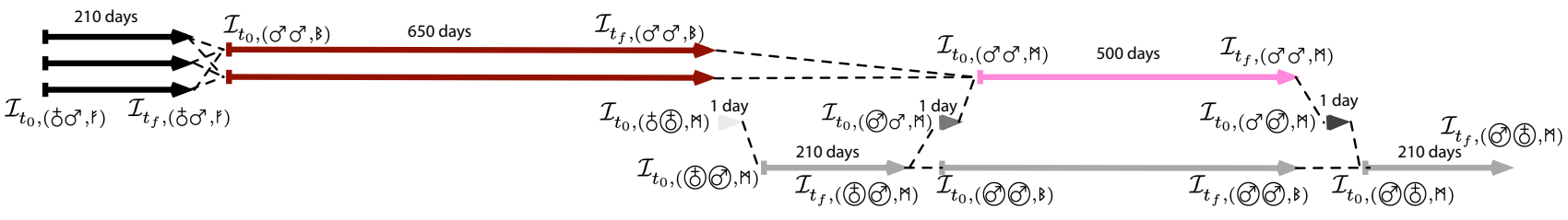

Figure 2. Modified ESM equation for Scenario 3 with terms decomposed by subsystem. Colored symbols for each location/subsystem represent ESM-specific elements and are placed beneath terms in the expanded equation to indicate dependence of the term to the element. Also provided is an timeline of inventory relationships colored by mission segment.

as grouped into functional categories. In our assumptions, we say that predeployment cargo is grouped into cargo shipments in set $j$ of $\mathcal{M}_{\mathrm{pd}_{j}}$ across some number of predeployment $n_{p d}$. We assume that this set of cargo is composed of items such as habitat assemblies, control hardware, photovoltaics \& batteries, reactors, tanks refrigerators, the various experimental apparatus, $3 \mathrm{D}$ printers, and other tools ${ }^{13}$. In the more expanded surface operations term, Figure 2 demonstrates that inventory for surface operations is composed of an assembled hab, process and reactor assemblies, mission crew, and integrated power systems. In this scenario, a set of equivalency factors are required for each segment of the mission. The equivalency factors for surface 
operations can be redefined in smaller terms of the habitat specifications rather than the larger values for rockets.

The location factor $L_{\mathrm{eq}}$ is the reciprocal of the payload fraction for transporting mass between two points in space and can be evaluated as the sum of across multiple orbital maneuvers with different $\Delta v$. Each element in the location mapping $\mathcal{L}_{2}$ has a specific required $\Delta v$. Any segment describing operations in a single location, such as Martian surface operations, has no mass transport and thus will have a $L_{\mathrm{eq}}=1.0$. Since $\Delta v$ can be related to the specific impulse $I_{\mathrm{sp}}$ and mass fraction $m_{0} / m_{f}$ via the Tsiolkovsky rocket equation ${ }^{1}$, we see how the mass of a specific segment inventory affects the location factor term. In terms of specific calculations, the mass fraction is the ratio of the of initial total rocket mass $m_{0}$ to final total mass $m_{f}$ and the payload fraction is the ratio of initial total mass $m_{0}$ to final delivered mass $m_{p}$ (no propellant, tanks, etc). Meanwhile, the $m_{0}$, $m_{f}$, and $m_{p}$ will be constrained by rocket technology choice. The scaling of location factor is nonlinear in the case where some number of predeployments are each limited in payload mass. We calculate the $\mathfrak{M}_{0, \mathrm{pd}}$ as the sum over the number of total predeployments $n_{\mathrm{pd}}$ where a given predeployment $j$ has a set of cargo $\mathcal{I}_{\mathrm{pd}_{j}}$ that doesn't require $V, P$, or $T$. The number of predeployment rockets will be parametric based on the $m_{p}$ for predeployment rockets and the sum of all inventory mass to be used on the martian surface shipped by predeployment. As shown in Figure 2, the $L_{\text {eq,pd }}$ in the $\mathfrak{M}_{0, \mathrm{pd}}$ term can be related to the $M$ and $V$ terms for the components of predeployment $j$ while the $L_{\mathrm{eq}, \mathrm{tr}_{1}}$ and $L_{\mathrm{eq}, \mathrm{tr}_{2}}$ terms are related to the $M$ and $V$ for all cargo transported in the complete mission.

Like $L_{\text {eq }}$, equivalency factors are also parametric based on certain elements of a segment inventory as showed by the colored markers in Figure 2. The volume equivalency $V_{\text {eq }}$ for crewed transits in space will be based on the pressurized volume ${ }^{54,59}$ of the vehicle. As opposed to just adopting constants that are not specific to the problem at hand, our framework provides the notation for specification of equivalencies based on their relationship to decision variables within the scope of the complete mission. For example, the mass of structure for some vehicle $m_{s}$ can be calculated in relation to the payload mass $m_{p}$ and the empty mass of a rocket $m_{e}$, providing a relationship of $m_{s}=m_{e}-m_{p}{ }^{60}$. The colors and symbols in Figure 2 provide visual insight into the relationships between how various inventory between segments can affect terms of the xESM equation when represented as the sum of segments. For example, $P_{\text {eq }}$ for surface operations will be dependent on the mass and power of the power generation hardware transported. These simple bounds illustrate the equivalency factors for one segment will often be parametrically related to decision variables in other segments. This realization only enforces the importance of our extension by which multiple segments are represented by a single optimization metric.

\section{Importance For Biomanufacturing-Based Missions}

To illustrate the process for calculating ESM with both the traditional approach and our proposed method, we provide the following example problem that has been scoped to the food production using Controlled Ecological Life Support Systems $(\text { CELSS })^{61,62}$, which we feel serves as an establish and graspable biomanufacturing-based technology ${ }^{48}$ for comparison against 'bring-everything' or physical/chemical life support systems ${ }^{63}$. Our example is defined for an ersatz mission of $6 \mathrm{CM}$ with 500 sols of surface operations flanked by interplanetary transits totaling 420 days ${ }^{50}$ with a simplified set of scenarios described in Table 1. The three scenarios compare the impact of agricultural biotechnology. Scenarios 2 and 3 adds distinction in the

\begin{tabular}{llll}
\hline Scenario & Predeployment & Transit & Surface Operations \\
\hline 1 & Send Nothing & Biomanufacturing System & 500 Days Operation \\
2 & Send Nothing & N/a & No Biomanufacturing \\
3 & Biomanufacturing System & N/a & 500 Days Operation \\
\hline
\end{tabular}

Table 1. Food system example ESM comparison.

decision to transport the biomanufacturing system in as predeployment or with the crew. During initial transit as well as return transit, the crew relies on prepackaged food. Crop growth begins on the initial day of surface operations. In the standard method, the surface operation constraints require that $\sim 50$ days of food would need to have been predeployed while the surface hardware grows the first crop because the constraints that specify surface operations have no input from transit ${ }^{13}$.

In cases where inventory from one segment can be used to satisfy constraints in another segment, the ESM summation of separately optimized mission segments can be less optimal than an ESM optimized with an objective function that accounts for both segments and constraint functions containing both terms from both segments. Thus, the goal of mathematically describing an optimized mission must be such that the objective function includes all mission segments and can be satisfied by solutions to constraints of coupled/related segments. Such description is not explicitly stated in any literature, although, the exact nature of the use of ESM is a matter of opinion ${ }^{64}$. The notation from previous ESM literature does not explicitly account for this, yet xESM provides the notation (and thus the pathway for corresponding computational methods) to account for constraints that may be satisfied from inventory across mission segments. In the following calculation, we explore two different themes for 
supplying food to astronauts, and the resulting ESM calculations from the standard approach compared to our proposed method for scenarios described in Table 1 using location and equivalency factors given in Table 2.

\begin{tabular}{llllllll}
\hline Segment & $\begin{array}{l}L_{\mathbf{e q}} \\
{[\mathbf{k g} / \mathbf{k g}]}\end{array}$ & $\begin{array}{l}V_{\mathbf{e q}} \\
{\left[\mathbf{k g} / \mathbf{m}^{\wedge} \mathbf{3}\right]}\end{array}$ & $\begin{array}{l}P_{\mathbf{e q}} \\
{[\mathbf{k g} / \mathbf{k W}]}\end{array}$ & $\begin{array}{l}C_{\mathbf{e q}} \\
{[\mathbf{k g} / \mathbf{k W}]}\end{array}$ & $T_{\mathrm{eq}}$ & $\begin{array}{l}\text { Number } \\
\text { of Days }\end{array}$ & $\begin{array}{l}\text { Number of } \\
\text { days food } \\
\text { eaten }\end{array}$ \\
\hline $\mathcal{M}_{\mathrm{pd}}$ & 2.77 & 9.16 & 237 & 40 & 0.7 & 210 & 0 \\
$\mathcal{M}_{\mathrm{tr}}$ & 10 & 133.8 & 136 & 50 & 0.7 & 410 & 410 \\
$\mathcal{M}_{\mathrm{sf}}$ & 1 & 9.16 & 228 & 145 & 0.7 & 500 & 500 \\
\hline
\end{tabular}

Table 2. Food system example location and equivalency factors.

In our example, we assume 6 crewmembers $(\mathrm{CM})$, each with a daily dry mass food requirement of $0.617 \mathrm{~kg} / \mathrm{CM}-\mathrm{d}^{13}$. We use this requirement to calculate the prepackaged food requirements of the two transit legs of each mission scenario, as well as the extra 50 or 500 days of food for surface operations in Scenarios 1 and 2 respectively. Given published infrastructure costs ${ }^{13}$ associated with a Mars Surface Habitat Vehicle ${ }^{20}$, we calculate ESM through consideration of the food subsystem including food, packaging, refrigeration ${ }^{13,20}$, and processing. Compare this case to a long-duration mission scenario in which food is grown during surface operations, in which literature suggests that a sizable initial hardware set would be required ${ }^{13}$. This set could include hydroponic growth chambers, water filtration, refrigeration, etc. along with additional support hardware like pumps, filters, etc ${ }^{13}$. For Scenarios 1 and 3 in our example problem, which utilize biomanufacturing, we include plant growth chamber infrasturcture and crop storage.

\begin{tabular}{lllllll}
\hline Scenario & Method & $\mathcal{M}_{\text {pd }}$ & $\mathcal{M}_{\text {tr }_{1}}$ & $\mathcal{M}_{\text {sf }}$ & $\mathcal{M}_{\text {tr }_{2}}$ & $\begin{array}{l}\text { Total } \\
{[\mathrm{kg}]}\end{array}$ \\
\hline \multirow{2}{*}{2} & Ext. ESM & 0 & 56059 & 2285 & 38547 & $\mathbf{9 6 8 9 2}$ \\
2 & Std. ESM & 0 & 56059 & 5889 & 38547 & 100496 \\
& Ext. ESM & 0 & 96145 & 781 & 38547 & $\mathbf{1 3 5 4 7 5}$ \\
3 & Std. ESM & 0 & 96145 & 8113 & 38547 & 142807 \\
& Ext. ESM & 1105 & 52067 & 2285 & 38547 & $\mathbf{9 4 0 0 6}$ \\
& Std. ESM & 1105 & 52067 & 5889 & 38547 & 97610 \\
\hline
\end{tabular}

Table 3. Food system example ESM comparison.

The comparison of the standard with XESM given in Table 3 show that XESM calculations show a reduction in ESM totals across mission segments, demonstrating that our proposed extension of the ESM allows for a more favorable representation of the impact on these factors over long-term missions. For Scenario 1, the use of xESM leads to 3.7\% ESM savings. For Scenario 2, the use of xESM leads to 5.4\% ESM savings. For Scenario 3, the use of xESM leads to 3.8\% ESM savings. These savings dramatically increase when including essential infrastructure from across all subsystems. Although, we clarify that the aim in exploring this example is not to make claims about specific technology, but rather to demonstrate an ESM cost reduction using the extended formulation.

\section{Towards ESM Minimization Under Uncertainty}

The aforementioned ESM definitions lead to the construction of a framework which should be complemented with an uncertainty modeling approach to make any related analysis robust. The xESM metric is ultimately determined based on some set of specific technologies that are used and whose operation is described by mathematical models. Thus, for each individual scenario (e.g., biologically-driven manufacturing) the xESM framework can be used both to analyze the cost of individual processes as well as the cost of integrated processes. Parts of the costs not commonly accounted for in cost calculations for space missions like ESM are uncertainty and risk. Risks can be broken down categorically into two groups: aleatory ${ }^{65}$ and epistemic ${ }^{66}$. Aleatory uncertainties are random and stochastic in nature and, although they can be examined via systematic testing, our empirical predictive confidence about their randomness cannot reduced below a threshold. On the other hand, epistemic uncertainties can be reduced through applying additional knowledge and testing, much more effectively. Both categories, though, can incorporated to the mathematical models that are utilized for system analysis and optimization. Therefore, models that describe the uncertainty in the various processes and operations that are carried out during each mission stage should be employed. 
Subsequently, the consideration of uncertainty leads to the quantification of reliability and risk. We are interested in quantifying the probability that the actual system operates as intended based on our best approximation of its behavior and the effect that disturbances will have on it. Thus, a binary classification of operational modes, i.e., failure or success mode, is typically employed in reliability engineering problems ${ }^{67}$. The aforementioned is, of course, a broad definition of success and failure of a task and needs to be contextualized for any scenario examined.

Here we discuss some possible direction to address the problem of optimal mission design under uncertainty, based on these ideas. As the entire mission is broken down in segments and sub-segments, we can define the tasks that have to fail (i.e., reach a failure mode based on some mathematical model) on particular time points during mission, so that the entire operation is considered to be failing. In other words, uncertainty can lead to a sequence of faults manifesting themselves until the mission has to be abandoned. This is a useful definition for incorporating risk into the mission design given the dynamic nature of operations and the breakdown of mission stages that was introduced earlier. Hence, taking the risk analysis into account, we are able to start defining a stochastic optimization problem ${ }^{68,69}$ for the mission design. In that case, the ESM is effectively a cost function tailored to account for multi-stage missions but not factoring in a penalty from failure. The goal is to use mathematical models and discover the optimal decisions that will lead to a design such that the mission objectives are fullfiled with the minimum possible cost. Process faults that can lead into a total failure can be taken into account and mitigated either by formulating a constrained cost minimization problem or by defining a penalty function that is added to the cost in case failure occurs given a set of decision variables/design. In both cases, either by using constraints or a simple penalty term, an overall ESM penalty can be quantified to describe how much the optimal decisions violate the success requirements. Under the simple assumptions that (1) the goal of human exploration missions is to carry out science experiments ${ }^{50}$ and that (2) experiments are carried out each day, a worst case scenario is a complete mission scrub in which all science objectives planned beyond the day of mission failure cannot be completed. Overall, the main idea is that if the mission is to fail on the very first days, then it would need to be redone on a following mission. This would practically cause the cost of the new mission to be augmented by at least the cost of the previous mission. The formulation of the minimization problem should be done such that part of the ESM cost of that mission left incomplete would be added onto next one. We argue that this is a valid initial construction of a constraint set or penalty term, based on assumption that incomplete work during a mission is required. This statement is especially valid for early human exploration missions where experimental use of new equipment is important in validating its use or raising technology readiness level to acceptable values for future missions. While we recognize that the standard recommendation in Decision Theory is to ignore sunk costs, we argue that the in our paradigm, this added penalty is not such a sunk cost. Given the assumption that initial exploration of Mars requires a set of experiments on planet in order to evaluate some set of technology platforms, if a mission fails to produce data requisite for some predefined validation because the mission is cut short, then those experiments will need to be re-run on a proceeding mission. In classical decision analysis, a sunk cost is a sum paid in the past that is no longer relevant to decisions in the future ${ }^{70}$ and thus should be ignored when making decisions. We argue that in our paradigm, we are analyzing the impact on a mission of some choice in technology that has some defined uncertainty, and thus no cost has been sunk. Instead, our paradigm is more in line with the concept of calculating the cost of some endeavor given the likelihood of failure, and thus making a decision based on the cost of needing to try something some number of times. In the parlance of decision analysis, this is an example of a prospective cost, and is not to be ignored. While simple, we argue that this assumption is valid as a modeling technique. It would be possible to draw the conclusion that we claim that the value of a mission depends on its duration, and thus a mission where life support fails for the last week is nearly as valuable as the full mission. We make no such claim but we do admit that such an extension can be drawn from a simple penalization of the cost.

\section{Future Work}

The use of the xESM framework helps guide the development and implementation of software for a biologically-driven reference mission architecture for long-duration human exploration of Mars. We recognize that this extension of ESM as a metric for mission scenario comparison is preliminary and not exhaustive in its scope. In addition to incorporation of mission parameters, specific constants and terms in our formulation are required, such as a more precise calculation of equivalency factors for cooling, power, volume, and crew-time and distillation of the specifics for risk fractions. Future endeavors include a comprehensive optimization problem formulation and solution based on the XESM framework both for biologically and non-biologically driven missions. Moving forward, we hope that our extension of ESM provides the basis for continued systems engineering and analysis research for a more quantitative and inclusive design and optimization of long term human exploration missions.

\section{References}

1. Wertz, J. R., Everett, D. F. \& Puschell, J. J. Space mission engineering: the new SMAD (Microcosm Press, 2011). 
2. Jones, H. W. Impact of Lower Launch Cost on Space Life Support. In 2018 AIAA SPACE and Astronautics Forum and Exposition, 5286 (2018).

3. Council, N. R. Advanced technology for human support in space (National Academies Press, 1997).

4. Drysdale, A. E. The effect of resource cost on life support selection. SAE Tech. Pap. 951492, 25 (1995).

5. Drysdale, A. Metrics and system analysis. Tech. Rep. (1998).

6. Levri, J. A., Vaccari, D. A. \& Drysdale, A. E. Theory and application of the equivalent system mass metric. Tech. Rep. (2000).

7. Messerschmid, E. \& Bertrand, R. Environmental control and life support system. In Space Stations, 109-145 (Springer, 1999).

8. Ono, E. \& Cuello, J. L. Photosynthetically Active Radiation (PAR) on Mars for Advanced Life Support. Tech. Rep. (2000).

9. Krumins, V., Strayer, R. \& Hummerick, M. Development of a fixed-film bioreactor for recycling of inedible plant nutrients in controlled biological systems. In 2001 ASAE Annual Meeting, 1 (American Society of Agricultural and Biological Engineers, 1998).

10. Hogan, J. et al. A Simulation Study Comparing Incineration and Composting in a Mars-Based Advanced Life Support System. (2000).

11. Morrow, R. C. \& Remiker, R. W. A deployable salad crop production system for lunar habitats. Tech. Rep. (2009).

12. Abney, M. B. et al. Ongoing Development of a Series Bosch Reactor System. In 43rd International Conference on Environmental Systems, 3512 (2013).

13. Anderson, M. S., Ewert, M. K. \& Keener, J. F. Life support baseline values and assumptions document. (2018).

14. Drysdale, A., Nakamura, T., Yorio, N., Sager, J. \& Wheeler, R. Use of sunlight for plant lighting in a bioregenerative life support system-equivalent system mass calculations. Adv. Space Res. 42, 1929-1943 (2008).

15. Swickrath, M., Anderson, M. \& Bagdigian, R. Parametric analysis of life support systems for future space exploration missions. In 41 st International Conference on Environmental Systems, 5039 (2011).

16. Sauser, B., Magnaye, R., Tan, W., Ramirez-Marquez, J. E. \& Sauser, B. Optimization of System Maturity and Equivalent System Mass for Space Systems Engineering Management. Tech. Rep. (2010).

17. Levri, J. et al. Food system trade study for an early Mars mission. Tech. Rep. (2001).

18. Flynn, M. et al. Planetary Water Recycling Systems Trade Study. In 49th International Conference on Environmental Systems (2019).

19. Feigel, A. Advancement of a Trade-off Tool for Life Support Technologies and its Application in Proposing a Life Support Architecture for the Gateway. (2019).

20. Yeh, H. Y. J., Brown, C. B., Anderson, M. S., Ewert, M. K. \& Jeng, F. F. ALSSAT development status. Tech. Rep. (2009).

21. Yeh, H. Y., Brown, C. B., Jeng, F. F., Lin, C. H. \& Ewert, M. K. ALSSAT Development Status and its Applications in Trade Studies. (2004).

22. Yeh, H. Y. J., Jeng, F. F., Brown, C. B., Lin, C. H. \& Ewert, M. K. Advanced Life Support Sizing Analysis Tool (ALSSAT) Using Microsoft@ Excel. Tech. Rep. (2001).

23. Czupalla, M., Zhukov, A., Mecsaci, A., Beck, M. \& Deiml, M. Dynamic life support system simulations with the virtual habitat. In 41 st International Conference on Environmental Systems, 5038 (2011).

24. Detrell, G., Belz, S. \& Keppler, J. ELISSA: A Life Support System (LSS) technology selection, modelling and simulation tool for human spaceflight missions. In 42nd COSPAR Scientific Assembly, vol. 42 (2018).

25. Olthoff, C., Pütz, D. \& Schnaitmann, J. Dynamic Life Support System Simulations with V-HAB (Deutsche Gesellschaft für Luft-und Raumfahrt-Lilienthal-Oberth eV, 2015).

26. Levri, J. et al. Advanced life support equivalent system mass guidelines document. (2003).

27. Escobar, C., Nabity, J. \& Klaus, D. Defining ECLSS Robustness for Deep Space Exploration (47th International Conference on Environmental Systems, 2017).

28. Detrell, G., Messerschmid, E. \& Ponsati, E. G. ECLSS reliability analysis tool for long duration spaceflight. In 46th International Conference on Environmental Systems (2016). 
29. Detrell, G. \& Belz, S. ELISSA-a comprehensive software package for ECLSS technology selection, modelling and simulation for human spaceflight missions (47th International Conference on Environmental Systems, 2017).

30. Curley, S., Stambaugh, I., Swickrath, M., Anderson, M. \& Rotter, H. Deep space habitat ECLSS design concept. In 42nd International Conference on Environmental Systems, 3417 (2012).

31. Peterson, L. J. Environmental Control and Life Support System (ECLSS) System Engineering Workshop. (2009).

32. Jones, H. Equivalent mass versus life cycle cost for life support technology selection. Tech. Rep. (2003).

33. Abney, M. B. et al. Comparison of Exploration Oxygen Recovery Technology Options Using ESM and LSMAC. In 49th International Conference on Environmental Systems (2020).

34. Jones, H. Multiple Metrics for Advanced Life Support. Tech. Rep. (1999).

35. Jones, H. W. \& Rasky, D. J. Advanced Life Support System Value Metric. (1999).

36. Jones, H. W. The System Complexity Metric (SCM) Predicts System Costs and Failure Rates. In 49th International Conference on Environmental Systems (2020).

37. Ho, K., De Weck, O. L., Hoffman, J. A. \& Shishko, R. Dynamic modeling and optimization for space logistics using time-expanded networks. Acta Astronaut. 105, 428-443 (2014).

38. Ishimatsu, T., Grogan, P. \& de Weck, O. Interplanetary Trajectory Analysis and Logistical Considerations of Human Mars Exploration. J. Cosmol. 12, 3588-3600 (2010).

39. Lee, G. et al. SpaceNet: Modeling and Simulating Space Logistics. In AIAA SPACE 2008 Conference \& Exposition, 7747 (2008).

40. Grogan, P., De Weck, O. \& Lee, C. Comparative usability study of two space logistics analysis tools. In AIAA SPACE 2011 Conference \& Exposition, 7345 (2011).

41. Grogan, P. T., Siddiqi, A. \& De Weck, O. L. Matrix methods for optimal manifesting of multinode space exploration systems. J. Spacecr. Rocket. 48, 679-690 (2011).

42. Do, S., Owens, A. \& Weck, O. d. HabNet-An Integrated Habitation and Supportability Architecting and Analysis Environment (45th International Conference on Environmental Systems, 2015).

43. Gill, T. R. Expanding Human Presence into the Solar System Starting with the Lunar Gateway. (2019).

44. Smith, M. et al. The Artemis Program: An Overview of NASA's Activities to Return Humans to the Moon. In 2020 IEEE Aerospace Conference, 1-10 (IEEE, 2020).

45. NASA's Lunar Exploration Program Overview. Tech. Rep. (2020).

46. Menezes, A. A., Cumbers, J., Hogan, J. A. \& Arkin, A. P. Towards synthetic biological approaches to resource utilization on space missions. J. The Royal Soc. Interface 12, 20140715 (2015).

47. Nangle, S. N. et al. The case for biotech on Mars. Nat. Biotechnol. 38, 401-407 (2020).

48. Berliner, A. et al. Towards a Biomanufactory on Mars. (2020).

49. Zubrin, R. M. \& Baker, D. A. Mars direct: humans to the red planet by 1999. Acta Astronaut. 26, 899-912 (1992).

50. Drake, B. G. \& Watts, K. D. Human Exploration of Mars Design Reference Architecture 5.0 Addendum\# 2. NASA. Tech. Rep. (2014).

51. Musk, E. Making Humans a Multi-Planetary Species. New Space 5, 46-61 (2017).

52. Musk, E. Making life multi-planetary. New Space 6, 2-11 (2018).

53. Reddy, V. S. The spacex effect. New Space 6, 125-134 (2018).

54. Fisher, J. W., Levri, J. A. \& Jones, H. W. The Effect of Mission Location on Mission Costs and Equivalent System Mass. Tech. Rep. (2003).

55. Thorsson, E. Futhark: A handbook of rune magic (Weiser Books, 2020).

56. Jones, H. Much Lower Launch Costs Make Resupply Cheaper Than Recycling for Space Life Support (47th International Conference on Environmental Systems, 2017).

57. Jones, H. The recent large reduction in space launch cost (48th International Conference on Environmental Systems, 2018).

58. Jones, H. W. The cost and equivalent system mass of space crew time. Tech. Rep. (2001).

59. Drysdale, A. E., Ewert, M. \& Hanford, A. J. Equivalent system mass studies of missions and concepts. Tech. Rep. (1999). 
60. Benson, T. Rocket Mass Ratios.

61. Wheeler, R. M., Mackowiak, C. L., Sager, J. C., Knott, W. M. \& Berry, W. L. Proximate composition of CELSS crops grown in NASA's biomass production chamber. Adv. Space Res. 18, 43-47 (1996).

62. Averner, M. M. The NASA CELSS program. (1990).

63. Jones, H. Comparison of Bioregenerative and Physical/Chemical Life Support Systems. SAE Transactions 181-192 (2006).

64. Jones, H. W. Don't trust a management metric, especially in life support (44th International Conference on Environmental Systems, 2014).

65. Apostolakis, G. The distinction between aleatory and epistemic uncertainties is important: an example from the inclusion of aging effects into PSA. In Proceedings of PSA '99, International Topical Meeting on Probabilistic Safety Assessment, 135-142 (1999).

66. Dezfuli, H., Kelly, D., Smith, C., Vedros, K. \& Galyean, W. Bayesian inference for NASA probabilistic risk and reliability analysis. (2009).

67. Dubourg, V., Sudret, B. \& Deheeger, F. Metamodel-based importance sampling for structural reliability analysis. Probabilistic Eng. Mech. 33, 47-57, DOI: https://doi.org/10.1016/j.probengmech.2013.02.002 (2013).

68. Mesbah, A., Streif, S., Findeisen, R. \& Braatz, R. D. Stochastic nonlinear model predictive control with probabilistic constraints. In 2014 American Control Conference, 2413-2419, DOI: 10.1109/ACC.2014.6858851 (2014).

69. Mesbah, A. Stochastic model predictive control: An overview and perspectives for future research. IEEE Control. Syst. Mag. 36, 30-44 (2016).

70. Hussain, T. Engineering economics (Laxmi Publications, Ltd., 2010).

\section{Acknowledgements}

This material is based upon work supported by NASA under grant or cooperative agreement award number NNX17AJ31G. We thank H. Jones (NASA Ames), O. de Weck (MIT) for their initial review. We thank Professors A.P. Arkin, A. Mesbah, and D. Clark for insights that continue to shape the problem of interest.

\section{Authorship Contributions}

A.J.B. and G.M. developed the concept and took lead in manuscript writing. A.J.B, G.M., and A.H. carried out computations. All authors provided critical feedback and helped shape the research, analyses, and manuscript.

\section{Competing Interests}

The authors declare that there is no conflict of interest. 